\title{
ÉTUDE DE LA CHÉTOTAXIE LARVAIRE DU « COMPLEXE MACULIPENNIS» (DIPTERA-CULICIDAE) DANS LA RÉgION TOULOUSAINE
}

\author{
J. SUZZONI-BLATGER* et A. SEVIN**
}

RÉSUMÉ. L'ensemble de la population larvaire d'atroparous de la région toulousaine ne diffère pas significativement de celle de Montpellier (France) et du Portugal, mais par contre diffère de celle d'Allemagne et d'Angleterre. La population de typicus ne diffère pas de celle de Montpellier, mais diffère de celle de l'Albanie. Les auteurs constatent la valeur taxonomique médiocre de la chétotaxie larvaire, du moins dans les zones où coexistent atroparvus, typicus et messeae.

\section{Study of the larval chaetotaxy in the "maculipennis complex" (Diptera- Culicidae) around Toulouse (Haute-Garonne, France)}

SUMMARY. The atroparvus larval population on the whole is not significantly different from that of Montpellier (France) and Portugal; on the other hand there is a highly significant difference with the populations of Germany and England. The typicus population does not differ significantly from that of Montpellier, but there is a significant difference with the population of Albany. The authors show that the larval chetotaxy is a feeble character for the taxonomy, at least when atroparous, typicus and messeae coexist.

\section{Généralités}

Anopheles maculipennis est le principal vecteur du paludisme en Europe et sur le pourtour du Bassin méditerranéen. C'est un complexe " d'espèces naissantes * (" incipient species » selon Dobzhansky) (5) composé de plusieurs membres difficiles à distinguer morphologiquement, et chez lesquels l'hybridation est parfois possible et peut donner des produits fertiles $(1,4)$. Dans la région toulousaine, le " complexe maculipennis " est composé d'atroparvus, typicus, subalpinus et messeae (14). Ces espèces se différencient essentiellement, et avec plus ou moins de facilité, par la mor-

* Laboratoire de Parasitologie, Faculté de Médecine Toulouse-Purpan, 37, allées Jules-Guesde, F 31000 Toulouse.

** Centre d'Hémotypologie du CNRS, Hôpital Purpan, F 31000 Toulouse.

Accepté le 4 juin r982. 
phologie ovulaire, la chétotaxie larvaire, et depuis quelques années par la cytogénétique et les systèmes gène-enzyme. Au cours d'une enquête sur le " complexe maculipennis ", nous avons étudié plus de 8000 larves, et il nous a semblé que les résultats obtenus avec un échantillonnage d'une telle importance pouvaient être intéressants car ils reflètent assez bien la réalité de l'ensemble de la population locale d'atroparvus et de typicus.

\section{Matériel et méthodes}

A partir du mois de juin, les femelles sont prélevées chaque semaine dans les abris d'animaux domestiques (bergeries, étables, porcheries) et transportées au laboratoire où elles sont élevées individuellement. Les pontes obtenues sont examinées puis classées avant la mise en élevage. Au $4^{\mathrm{e}}$ stade, on compte les soies antépalmées des segments IV et V des larves. Seules sont conservées les souches (chaque souche étant issue d'une femelle mère) ayant plus de 30 larves de stade IV, nombre minimum pour une étude statistique portant sur de grands échantillons.

\section{Résultats}

Nous avons compté les soies antépalmées des segments IV et V de 8453 larves d'Anopheles maculipennis. Parmi celles-ci, nous en avons retenu 7738 provenant de souches ayant plus de 30 individus.

\section{1 - Atroparvus}

Sur 5421 larves, la moyenne des ramifications des soies antépalmées est $\bar{M}=$ $11,56 \pm 0,05$ (intervalle de confiance de la moyenne au seuil de $5 \%$ ) et l'écart-type $\sigma=2,04$. Les moyennes des différentes souches s'échelonnent de 8,56 $\pm 0,24(\mathrm{n}=45$ et $\sigma=0,81)$ à $14,43 \pm 0,75(\mathrm{n}=30$ et $\sigma=2,10)$. Les valeurs extrêmes des ramifications des soies sont 7 et 22. Sur 5471 larves, 84,32\% ont des soies dont les ramifications sont comprises entre 9 et 14. L'étude séparée des deux gîtes principaux a donné les résultats suivants : $\bar{M}=11,78 \pm 0,08(\mathrm{n}=2694$ et $\sigma=1,99)$ et $\bar{M}=11,35$ $\pm 0,08(\mathrm{n}=2727$ et $\sigma=2,07)$.

\section{2 - Typicus}

La moyenne est $\overline{\mathrm{M}}=13,74 \pm 0,10$ pour $\mathrm{n}=1723$ avec $\sigma=2,14$. Les moyenne $\mathrm{S}^{\mathrm{S}}$ des différentes souches obtenues s'échelonnent de $\bar{M}=12,07 \pm 0,21\left(\mathrm{n}=106 \mathrm{e}^{\mathrm{t}}\right.$ $\sigma=1,10)$ à $\overline{\mathrm{M}}=17,97 \pm 0,64(\mathrm{n}=31$ et $\sigma=1,82)$. Les valeurs extrêmes des brin $\mathrm{S}$ des soies sont 7 et 23. En étudiant séparément les deux gîtes principaux, on obtient pour l'un $\overline{\mathrm{M}}=13,74 \pm 0,11(\mathrm{n}=1485$ et $\sigma=2,17)$ et pour l'autre $\overline{\mathrm{M}}=13,73 \pm$ $0,25(\mathrm{n}=238$ et $\sigma=1,99)$. Sur 1852 larves, $84,93 \%$ possèdent entre 11 et 16 ramifications. 


\section{3 - Subalpinus}

L'espèce subalpinus n'a été trouvée à l'état adulte que dans une seule bergerie. La moyenne des soies antépalmées est $\bar{M}=19,29 \pm 0,33(\mathrm{n}=198)$. Les moyennes des trois souches obtenues sont : 18,07 $\pm 0,64(\mathrm{n}=75$ et $\sigma=2,81) ; 19,93 \pm 1,12$ $(\mathrm{n}=30$ et $\sigma=3,14) ; 20,08 \pm 0,49(\mathrm{n}=93$ et $\sigma=2,44)$. Les valeurs extrêmes des brins des soies sont 12 et 28. 46,46\% des larves ont des soies ayant de 20 à 28 ramifications et $57,57 \%$ en ont de 19 à 28 .

\section{4 - Messeae}

L'espèce messeae plusieurs fois pressentie dans la région de Toulouse a été déterminée pour la première fois en Haute-Garonne (14) en $1979: \overline{\mathrm{M}}=15,88 \pm 0,79$ ( $\mathrm{n}=27$ et $\sigma=2,10$ ). Depuis, elle n'a été retrouvée que deux fois dans deux gîtes différents : $\overline{\mathrm{M}}=18,10 \pm 0,44(\mathrm{n}=115$ et $\sigma=2,42)$. Les valeurs extrêmes des ramifications des soies sont 11 et 27 . Sur 142 individus, $50 \%$ ont des soies ayant entre 11 et 17 ramifications et $62,68 \%$ en ont entre 13 et 18 .

\section{Discussion}

Les soies antépalmées des segments IV et V des larves de $4^{\mathbf{e}}$ stade ont longtemps été considérées comme le meilleur caractère morphologique permettant la différenciation des membres du " complexe maculipennis " mais on s'est rendu compte depuis de la valeur très limitée de ce caractère. En effet, en utilisant toutes les données bibliographiques connues et résumées dans le tableau $I$, nous n'aurions pu déterminer que $71,6 \%$ des souches d'atroparvus et $42,3 \%$ des souches de typicus.

Le traitement statistique des données montre que l'ensemble de la population larvaire de la région toulousaine ne diffère pas significativement de celle de Montpellier (France) (7) $(\varepsilon=1,88$ avec un risque de $5 \%$ ) et du Portugal $(\varepsilon=0,73)$. Par contre, il existe une différence hautement significative avec la population larvaire d'atroparvus d'Allemagne $\left(\varepsilon=5,69\right.$ avec un risque $<$ à $\left.10^{-7}\right)$, et d'Angleterre $(\varepsilon=$ 8,16 avec un risque $<$ à $10^{-8}$ ) (1). Les données concernant ce dernier pays ne reflètent pas l'ensemble de la population anglaise car les larves proviennent de la souche Epsom issue d'une femelle unique (13).

L'ensemble de la population de typicus de Toulouse ne diffère pas significativement de celle de Montpellier $(\varepsilon=0,74$ au seuil de $5 \%$ ). Par contre, elle est significativement différente de celle de l'Albanie (2) $\left(\varepsilon=5,61\right.$ avec un risque $<$ à $\left.10^{-7}\right)$.

En ce qui concerne subalpinus malgré notre faible échantillonnage (198 larves), la population apparaît significativement différente de celle de Montpellier $(\varepsilon=17,12$ avec un risque $<$ à $\left.10^{-6}\right)$, et de celle de l'Albanie $\left(\varepsilon=9,34\right.$ avec un risque $<$ à $\left.10^{-6}\right)(8)$.

A cause de données insuffisantes, nous n'avons pas pu effectuer de comparaison statistique pour messeae. Toutefois, les moyennes des souches que nous avons obtenues 
TABLEAU I. - Récapitulation des valeurs des soies antépalmées fournies par la bibliographie pour l'étude du " complexe maculipennis "

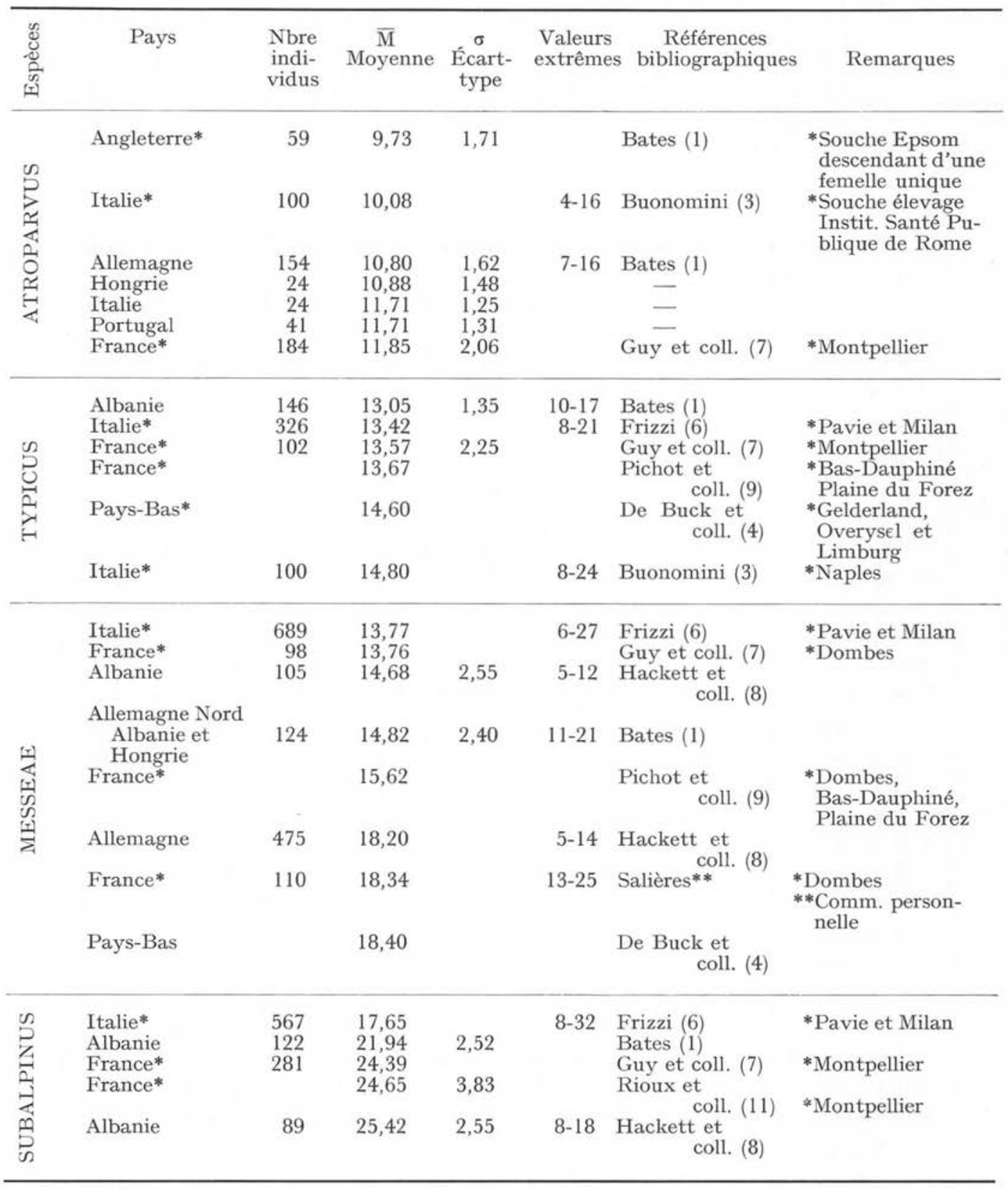


se rapprochent de celles des Dombes et de l'Allemagne, mais diffèrent nettement de celles de l'Albanie, pays où a été posé le problème de la confusion entre typicus et messeae.

Le chevauchement des nombres extrêmes des ramifications des soies est bien plus important que celui signalé par Bates en Europe (1) et par Salières et coll. (12) sur le littoral Languedoc-Roussillon.

\section{Conclusion}

L'étude de la chétotaxie larvaire d'Anopheles maculipennis montre qu'il n'existe pas de différence significative entre la population d'atroparvus de la région toulousaine et celle de Montpellier (France) et du Portugal ; par contre, il existe une différence hautement significative avec les populations larvaires d'Allemagne et d'Angleterre. La population de typicus ne diffère pas de celle de Montpellier, mais diffère de celle de l'Albanie. En ce qui concerne subalpinus et messeae, l'échantillonnage obtenu dans notre région nous paraît insuffisant, car la moyenne d'un échantillon est d'autant plus près de la moyenne réelle de la totalité de la population que l'échantillon est plus grand.

La chétotaxie larvaire est un caractère taxonomique assez médiocre permettant difficilement la différenciation d'atroparvus, de typicus et de messeae, du moins lorsque ces espèces coexistent dans les mêmes gîtes (3) ou dans des gîtes voisins. Le nombre des ramifications des soies antépalmées des larves du $4 \mathrm{e}$ stade paraît déterminé par des systèmes polygéniques. La suite de nos travaux (à publier prochainement) fait apparaitre que ce caractère varie de façon continue et qu'il n'existe pas de lignées pures pour un nombre déterminé de ramifications.

\section{BIBLIOGRAPHIE}

I. BAtes M. : Variation in the antepalmate hairs of larvae of the maculipennis complex. Riv. Malariol, 1939, 18, 299-312.

2. Bates M., Hacketт L. W. : The distinguishing characteristics of the populations of Anopheles maculipennis found in the Southern Europe. Verh. VII Intern. Kongress für Entomologie (1938), 1939, I555-I 569.

3. Buonomini G. : L'esame delle setole antepalmate per lo studio della popolazione larvale dei focolai naturali di $A$. maculipennis. Riv. Parassitol., 1940, 4, 163-174.

4. De Buck A., Schoute E., Swellengrebel N. H. : Crossbreeding experiments with dutch and foreing races of Anopheles maculipennis. Riv. Malariol., I934, 13, 237-263.

5. Doвzhansкy Th. : Génétique du processus évolutif, 1977. Flammarion, Paris, $5^{8} 3$ p.

6. Frizzi G. : Nuovi contributi e prospettive du ricerca nel gruppo Anopheles maculipennis in base allo studio del dimorfismo cromosomico (ordinamento ad $\mathrm{X}$ invertito e tipico) nel messeae, Pavie, $3^{e}$ symposia genetica, 1952, 3, 231-265.

7. GuY Y, Salieres A., Boesiger E. : Contribution à l'étude du "complexe maculipennis ". Mise au point en 1975. Ann. biol., 1976, Is, 227-282.

8. Hackett L. W., Lewis D. J. : A new variety of Anopheles maculipennis in southern Europe. Riv. Malariol., 1935, 14, 377-383.

9. Pichot J., Deruaz D. : Les anophèles du complexe maculipennis dans la région lyonnaise. Lyon Méd., 1981, 245, no h.s., II7-121. 
ro. Rioux J. A. : Les Culicides du "Midi méditerranéen ». Encycl. entomol., 35, 303 p., Lechevalier, Paris, 1958.

II. Rioux J. A., Ruffie J. : Présence d'Anopheles maculipennis s. sp. subalpinus Hackett et Lewis I937 en Bas-Languedoc. Intérêt des méthodes caryosystématique et statistique. Bull. Soc. Pathol. Exot., I957, so, 831-837.

i2. Salieres A., Guy Y., Suzzoni-Blatger J., Cousserans J. : Bilan de quatre années de recherches sur le "complexe maculipennis » (Diptera-Culicidae-Anophelinae). Ann. Parasitol. Hum. Comp., 1978, 53, 751-756.

13. Shute P. G., Ungureanu E. : Comparative studies of the eggs of Anopheles maculipennis var. atroparous in the field and in the laboratory. Riv. Malariol., 1938, $17,35^{8-36 \mathrm{I}}$.

14. Suzzoni-Blatger J., Anglade F., Larrouy G. : Présence d'Anopheles messeae (DipteraCulicidae) en Haute-Garonne. Bull. Soc. Hist. Nat., Toulouse, 1980, 1 16, 175-178.

Des Microfiches et des Microfilms de Cette PUblication PEUVENT Être obtenUS AUPRÈS DE:

This publication is available in Microform from :

MASSON - SPPIF (réf. MIMC)

I20, boulevard Saint-Germain, 75280 PARIS CEDEX 06

Masson, Paris, 1982.

Le Directeur de la Publication : Dr J. TALAMon

Tous droits de traduction, d'adaptation et de reproduction par tous procédés pour tous pays

La loi du II mars I957 n'autorisant, aux termes des alinéas 2 et 3 de l'article 4I, d'une part, que les "copies ou reproductions strictement réservées à l'usage privé du copiste et non destinées à une utilisation collective " et, d'autre part, que les analyses et les courtes citations dans un but d'exemple et d'illustration, " toute représentation ou reproduction intégrale ou partielle, faite sans le consentement de l'auteur ou de ses ayants droit ou ayants cause, est illicite „ (alinéa I Ier de l'article 40).

Cette représentation ou reproduction, par quelque procédé que ce soit, constituerait donc une contrefaçon sanctionnée par les articles 425 et suivants du Code pénal.

Masson, éditeur, Paris. - Dépôt légal : I982 - No d'ordre : 5424. - janvier I983

IMPRIMERIE DARANTIERE DIJON-QUETIGNY (FRANCE). -

Commission paritaire $n^{0} 54169$

Printed in France. 